\title{
Selective adaptation effects on end-point stimuli in a speech series
}

\author{
JAMLS R. SAWUSCH \\ Indiana University, Bloomington, Indiana 4740 I
}

\begin{abstract}
Recent experiments in speech perception using the selective adaptation paradigm have found that the phonetic boundary of a test series shifts following adaptation. However, no changes within the phonetic category have been found. In the present experiment, a series of voiced CV syllables which varied along the feature of place was used in a selective adaptation paradigm. The end-point stimuli from the test series were used as adaptors. Subjects used a 6-point rating scale to respond to the stimuli instead of the usual two-category identification. The average rating for end-point stimuli from the same category as the adaptor, as well as the boundary stimuli, shifted as a function of adaptation. In all cases, the average rating response shifted toward that of the unadapted category. The average rating for stimuli in the opposite category from that of the adaptor remained relatively unchanged. These results indicate that the entire category of the adapting stimulus changes as a function of selective adaptation and that the effect is not confined to stimuli near the phonetic boundary.
\end{abstract}

Recent models of the speech perception system have been proposed within an information processing framework. These models have generally proposed successive levels of auditory and phonetic analysis of the speech waveform (Cooper, 1975; Pisoni, 1975; Pisoni \& Sawusch, 1975; Tartter \& Eimas, 1975). Although there has been some consensus on the distinction between auditory and phonetic levels of processing, the precise nature of the perceptual mechanism at each of these levels is still under intensive investigation. Abbs and Sussman (1971) proposed that models of speech perception be phrased in terms of selectively tuned feature detectors. Specifically, they suggested that these feature detectors would be tuned to such types of information as a change of frequency, high energy concentration at low frequencies, and so forth (Abbs \& Sussman, 1971). These types of information have been found to be sufficient cues for the identification of certain speech sounds (see Liberman, Cooper, Shankweiler, \& Studdert-Kennedy, 1967, for review).

Evidence implicating the existence of feature detectors was first reported by Eimas and his coworkers (Eimas, Cooper \& Corbit, 1973; Eimas \& Corbit, 1973). Their studies, using the selective adaptation paradigm, demonstrated the presence of some form of feature detector in the perception of the voicing feature. Since these initial results, further research has implicated detector mechanisms in mediating the perception of the feature of place of production (Cooper, 1974a; Cooper \& Blumstein,

This research was supported by NIH Research Grant NS-12179-01 to Indiana University. I would like to thank David B. Pisoni for his insight and suggestions during all phases of this work and Dr. F.S. Cooper for making the facilities at Haskins laboratories available for stimulus preparation.
1974; Pisoni \& Tash, 1975; Tartter \& Eimas, 1975) as well as the voicing feature (Cooper, 1974b, c; Tartter \& Eimas, 1975).

Concurrent with these new experimental results, models of the perceptual system have been proposed to account for these findings. Among the models proposed, those of Cooper and Nager (1975) and Tartter and Eimas (1975) are fairly specific. Both models propose successive stages of auditory and phonetic analysis. Both models also assume that selective adaptation operates to fatigue detectors operating at both auditory and phonetic levels. The evidence for selective adaptation affecting detectors at an auditory level of analysis is fairly strong (Ades, 1974; Pisoni \& Tash, 1975; Tartter \& Eimas, 1975; Bailey, Note 1). The evidence for selective adaptation operating at a phonetic level has not been as strong, and interpretations of this evidence have diverged accordingly (Cooper, 1975; Pisoni \& Tash, 1975; Tartter \& Eimas, 1975).

Another assumption of both models is that selective adaptation is assumed to affect the entire range of a feature detector. However, except for a recent study by Miller (1975), there has been no direct empirical support for this assumption. The general result of selective adaptation studies is that following adaptation, the phonetic boundary shifts toward the category of the adaptor. This shift in identification is mirrored by a shift in discrimination (Cooper, 1974a). However, the shifts for both identification and discrimination are confined to stimuli close to the phonetic boundaries. No systematic changes have been observed at the end points of a test series for a standard adaptation paradigm.

Miller (1975) reasoned that no shift had been found near the end points of the test series because the 
identification paradigm was too insensitive. The subject's task in a standard identification paradigm is simply to categorize the stimuli using one of two or three phonetic labels. Miller (1975) used a dichotic listening paradigm and investigated feature competition among good examplars of stop CV syllables both before and after selective adaption. The results indicated that features were less effective in competing after adaptation. Thus, these results indicate that selective adaptation does affect the processing of good exemplars of a category and not merely boundary stimuli.

If Miller's explanation of the failure of identification studies to pick up end-point shifts in selective adaptation is correct, then it should be possible to pick up end-point effects with a more sensitive identification paradigm. Glanzman and Pisoni (Note 2) used confidence ratings in conjunction with $\mathrm{ABX}$ and 4IAX discrimination paradigms. Their results indicated that subjects' assignment of ratings was directly related to their accuracy of discrimination. In using confidence ratings, Glanzman and Pisoni tapped a source of information that previous discrimination experiments had neglected. Their results demonstrated that subjects could provide information about the stimuli beyond the simple discrimination response. From these results, it seemed plausible that the use of a rating scale, rather than a two-category judgment, would be a more sensitive measure in an identification paradigm.

The purpose of the present experiment was twofold. First, could subjects use a rating scale in an identification task consistently? If subjects could use the rating scale, then would other stimuli, besides those at the phonetic boundary, show an effect due to selective adaptation?

\section{METHOD}

\section{Subjects}

Fourteen paid volunteers served as subjects. All were righthanded native speakers of English with no known history of a hearing or speech disorder. The subjects responded to an advertisement in the student newspaper and were paid at the rate of $\$ 2 / \mathrm{h}$. The subjects were divided into two groups of seven subjects each.

\footnotetext{
Stimuli

The stimuli were three formant synthetic $\mathrm{CV}$ syllables that were prepared on the parallel resonance synthesizer at Haskins Laboratories. All stimuli were recorded on magnetic tape for later playback. The test stimuli consisted of one series of nine CV syllables that ranged perceptually from [bi] to [di] on the place feature. These stimuli varied in their starting frequencies for the second and third formant transitions. These initial frequencies are displayed in Table 1 . The duration of the formant transitions was $50 \mathrm{msec}$, followed by a $250-\mathrm{msec}$ steady state vowel [i]. The vowel had formant center frequencies of 287 , 2,307 , and $3,026 \mathrm{~Hz}$ for the first through third formants, respectively.
}

\section{Procedure}

The experimental tapes were reproduced on a high-quality tape
Table 1

Starting Frequencies of the Second and Third Formant Transitions for the Synthetic CV Series [bi] - [di]

\begin{tabular}{ccc}
$\begin{array}{c}\text { Stimulus } \\
\text { Number }\end{array}$ & \multicolumn{2}{c}{$\begin{array}{c}\text { Starting Frequencies (in } \mathrm{Hz}) \\
\mathrm{F}_{2}\end{array}$} \\
\hline 1 & 1465 & $\mathrm{~F}_{\mathbf{3}}$ \\
2 & 1541 & 2180 \\
3 & 1620 & 2348 \\
4 & 1695 & 2525 \\
5 & 1772 & 2694 \\
6 & 1845 & 2862 \\
7 & 1920 & 3026 \\
8 & 1996 & 3195 \\
9 & 2078 & 3363 \\
\hline
\end{tabular}

Note-The fixed steady-state formants were centered at: $286 \mathrm{~Hz}\left(F_{1}\right), 2,307 \mathrm{~Hz}\left(F_{2}\right)$, and 3,026 Hz$\left(F_{3}\right)$.

recorder (Ampex AG-500) and were presented binaurally through Telephonics (TDH-39) matched and calibrated headphones. The gain of the tape recorder was adjusted to give a voltage across the headphones equivalent to $80 \mathrm{~dB}$ SPL for a steady state calibration vowel [a].

The experiment was conducted on 2 consecutive days. At the beginning of each day, subjects listened to two identification tapes. Each tape contained a different randomization of 10 replications of each of the nine stimuli. Subjects were told that they would hear synthetic speech sounds approximating the syllables [bi] and [di]. They were instructed to respond to the stimuli using a 6-point rating scale. A copy of the response scale was present in front of each subject at all times. The rating scale is presented in Table 2 . The subjects recorded their rating responses in prepared response booklets.

Immediately following the identification tapes, an adaptation test was presented. One group of subjects received the adapting syllable [bi] and the other group received the syllable [di]. The adapting stimuli were the end-point syllables from the test series. The adapting syllable was presented for $1 \mathrm{~min}$ (100 repetitions with a 300 -msec interstimulus interval). After each minute of adaptation, the nine test syllables were presented in random order for identification by subjects using the rating scale. Nine of these adaptation trials were run on each day. Thus, at the end of the experiment, each subject had furnished 18 adapted responses to each of the nine stimuli.

\section{RESULTS \& DISCUSSION}

The data from the first identification test tape on the first day were treated as practice runs for subjects to accustom themselves to the rating scale. These data were eliminated from further analysis for all subjects.

The identification data for both groups is pooled together in Figure 1. Subjects systematically assigned

Table 2

Six-Point Rating Scale that Subjects Used in Responding to the Test Stimuli

\begin{tabular}{cl}
\hline Response & \multicolumn{1}{c}{ Rating } \\
\hline 1 & Positive syllable was a "bi" \\
2 & Syllable sounded like a "bi" \\
3 & Unsure, syllable could have been a "bi" \\
4 & Unsure, syllable could have been a "di" \\
5 & Syllable sounded like a "di" \\
6 & Positive syllable was a "di" \\
\hline
\end{tabular}




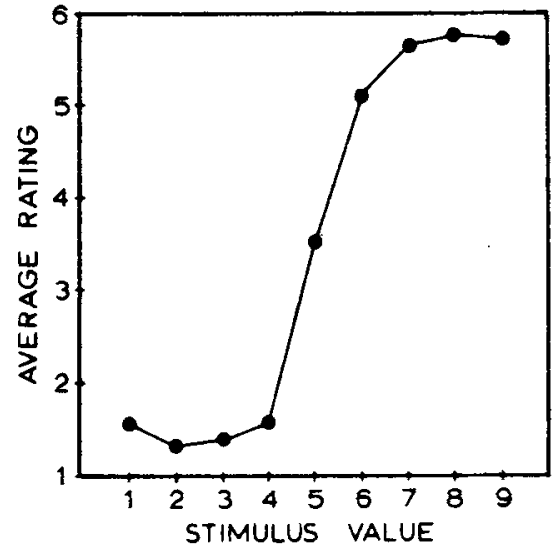

Figure 1. The average rating response of all 14 subjects from Groups 1 and 2 for the unadapted [bi]-[di] series.

low ratings to the stimuli at the [bi] end of the series (Stimuli 1-4) and high ratings to the stimuli at the [di] end of the series (Stimuli 6-9). Stimulus 5 received an ambiguous rating of approximately 3.50. One other point to note is the upturn in the ratings at Stimulus 1. It appears that the best exemplars of the [bi] category are Stimuli 2 and 3. Stimulus 1 received a significantly higher rating than Stimulus 2 $[t(13)=2.654, p<.01$, for a one-tailed, correlated $t$ test]. Thus, it appears that subjects can provide a reliable rating response in an identification paradigm. Further, the rating response appears to be more sensitive to differences between stimuli from the same phonetic category than the standard identification paradigm.

In order to compare the adaptation data from the present experiment with previous selective adaptation experiments, the 6-point rating scale data was collapsed into a two-category scale. Responses 1,2 , and 3 were treated as "bi" responses. Similarly, Responses 4, 5, and 6 were treated as "di" responses. The data for both groups, before and after adaptation, is plotted in Figure 2 as percent of "bi" responses. The shifts in the phonetic category boundaries for both groups were highly significant $[t(6)=5.14$, $p<.005$, for the [bi] adapted group; $t(6)=7.45$, $p<.001$, for the [di] adapted group using one-tailed, correlated $t$ tests]. These shifts are similar to those found previously for selective adaptation on a place series (Cooper, 1974a; Pisoni \& Tash, 1975; Tartter \& Eimas, 1975).

The average rating response for each of the nine stimuli before and after [bi] adaptation is displayed in the left-hand panel of Figure 3. Since individual subjects showed the same trend as the group, only the group data is shown. The shift in the average rating for each of the first five stimuli was significant $[t(6)=2.21, p<.05$, for Stimulus $1 ; t(6)=2.02$, $p<.05$, for Stimulus $2 ; t(6)=3.18, p<.01$, for Stimulus $3 ; t(6)=3.32, p<.01$, for Stimulus 4; and $t(6)=5.00, p<.005$, for Stimulus 5; all one- tailed, correlated $t$ tests]. The shifts in average rating for each of Stimuli 6 through 9 were not significant, and were not in the same direction for all subjects.

The right-hand panel of Figure 3 displays the average rating responses before and after adaptation for the [di] adapted group. The shifts in the average rating for each of the last six stimuli were significant $[t(6)=2.45, p<.05$, for Stimulus $4 ; t(6)=4.36$, $p<.005$, for Stimulus $5 ; t(6)=7.09, p<.001$, for Stimulus $6 ; t(6)=6.39, p<.001$, for Stimulus 7 ; $t(6)=5.61, p<.001$, for Stimulus 8; and $t(6)=4.16$, $p<.005$, for Stimulus 9, all one-tailed correlated $t$ tests]. The shifts in the average rating for each of Stimuli 1,2 and 3 were not significant, although they were generally in the same direction as the shifts for Stimuli 4 through 9.

These results clearly indicate that selective adaptation affects the entire category from which the adapting stimulus is drawn, and not just the boundary stimuli. Further, because the opposite category was relatively unaffected, two inferences can be drawn. First, any detector operating for the opposing category was unaffected by selective adaptation, and thus these detectors operate independently of each other. This can be contrasted with an "opponent process" model in which a strong output from one detector would inhibit the output of an opposing detector. In such a model, the ratings in the unadapted category should change due to adaptation. Specifically, following [bi] adaptation, the stimuli at the [di] end of the series (Stimuli 7, 8, and 9) should have

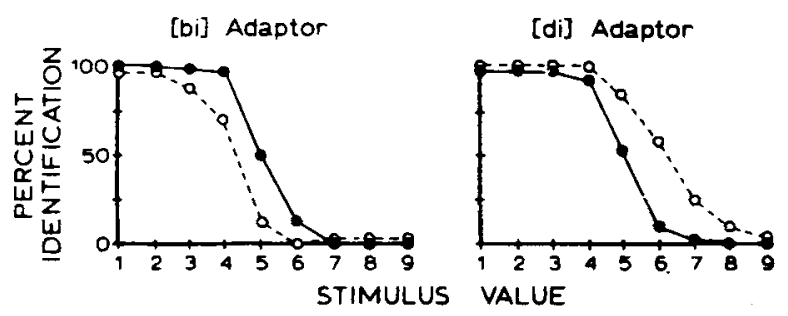

Figure 2. Unadapted (solid circles) and adapted (open circles) identification functions for the [bi]-[di] series. The six rating scale responses were collapsed into two-category responses.
GROUP I

[bi] Adaptor

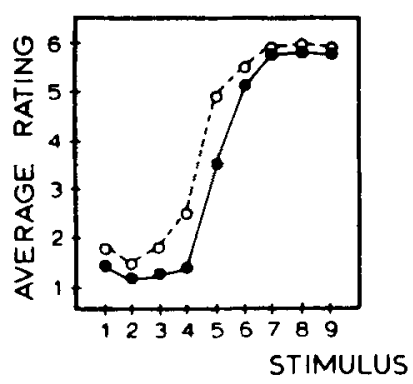

GROUP II

[di] Adaptor

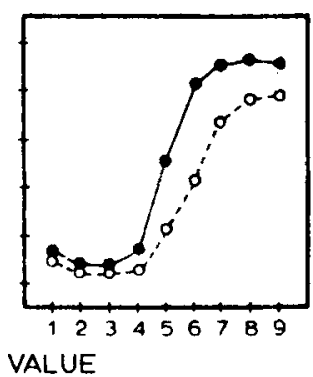

Figure 3. Unadapted (solid circles) and adapted (open circles) rating response functions. Group I $(N=7)$ received the [bi] adaptor and Group II ( $N=7)$ received the [di] adaptor. 
received higher (more positive di) ratings, and vice versa. This result was not found, and thus the opponent process model is not supported. Secondly, opposing detectors do not extend significantly into each other's range. Rather, their response range seems to be sharply delimited. If there were a significant degree of overlap between opposing detectors, then the rating responses should have changed in the unadapted, as well as the adapted, categories. The failure to find a consistent change in the unadapted category indicates that the opposing detectors do not overlap extensively.

These conclusions must, however, be tempered somewhat. There exists the possibility that no change occurred in the predicted direction within the unadapted categories because of a ceiling (or floor) effect. For example, the average rating before adaptation within the "bi" category was approximately 1.4 for the first four stimuli (for all subjects). If the actual effect of adaptation with the syllable [di] was to enhance "bi" responses, then the rating response would not be likely to show this, because a 1 is the lowest (most "bi" like) rating possible. Four of the seven subjects in each group exhibited a ceiling (floor) effect. However, three subjects in each group did not show ceiling or floor effects in the unadapted categories. The rating response shifts for these subjects, within the unadapted categories, were inconsistent. In each group, one subject showed a small shift in the unadapted category in the same direction as that of the adapted category. Another subject in each group exhibited a small shift in the unadapted category in the opposite direction to that of the adapted category. The third subject in each group showed no shift in the unadapted category. Since these subjects did not show ceiling (floor) effects, the lack of a consistent rating shift within the unadapted categories can be interpreted as support for the two inferences mentioned above.

This experiment does not answer the question as to the level at which selective adaptation takes place. The changes in rating response in the present experiment could be taking place entirely within an auditory level of processing (Pisoni \& Tash, 1975) or they could reflect changes at both auditory and phonetic levels (Cooper, 1975; Tartter \& Eimas, 1975). However, regardless of the processing level at which the adaptation effect takes place, information about the relative outputs of the detector mechanisms operating is preserved and transmitted to higher levels. If this type of information were not retained and used, then subjects would not have shown a change in their rating response as a function of adaptation and the rating responses for all stimuli from within the same phonetic category would have been identical.
In summary, the following conclusions seem justified in light of these results. First, subjects can effectively use a rating scale for responding in an identification task. Further, their responses indicate that all stimuli from within the same phonetic category are not treated equally. Information differentiating stimuli within a phonetic category is available to subjects, and they use this information in making a rating response. Secondly, the present results agree with those reported recently by Miller (1975). Selective adaptation affects the entire category from which the adapting stimulus is drawn, including good stimulus exemplars. The opposing category is relatively unaffected by the adaptation process. This is reflected in the change in subjects' ratings of stimuli from the same phonetic category as the adaptor. These results are consistent with feature detector models of speech perception which have assumed that selective adaptation affects the entire range of a detector (Cooper \& Nager, 1975; Tartter \& Eimas, 1975). Furthermore, the present results indicate that the detectors being affected operate independently of each other. An opponent process model of detector operation is inconsistent with the current results. Hopefully, further use of the rating scale in conjunction with selective adaptation paradigms will further illuminate the nature of the selective adaptation pheonomena and the more general process of speech perception.

\section{REFERENCE NOTES}

1. Bailey, P. Perceptual adaptation for acoustical features in speech. Speech Perception: Report on Research in Progress in the Department of Psychology. Queen's University of Belfast, 1973, 2.2, 29-34.

2. Glanzman, D. L., \& Pisoni, D. B. Decision processes in speech discrimination as revealed by confidence ratings. Paper presented at the 85th meeting of the Acoustical Society of America. Boston. April 1973.

\section{REFERENCES}

Abbs, J. H., \& Sussman. H. M. Neurophysiological feature detectors and speech perception: A discussion of theoretical implications. Journal of Speech and Hearing Research. 1971, 14, 23-36.

ADEs, A. E. How phonetic is selective adaptation? Experiments on syllable position and vowel environment. Perception \& Psychophysics, 1974, 16, 61-67.

COOPER, W. E. Adaptation of phonetic feature analyzers for place of articulation. Joumal of the Acoustical Society of America, 1974, 56, 617-627; (a)

Cooper, W. E. Contingent feature analysis in speech perception. Perception \& Psychophysics, 1974, 16, 201-204. (b)

CoOper, W, E. Selective adaptation for acoustic cues of voicing in initial stops. Journal of Phonetics, 1974, 2, 303-313. (c)

Cooper, W. E. Selective adaptation to speech. In F. Restle, R. M. Shiffrin, N. J. Castellan, H. Lindman, and D. B. 
Pisoni (Eds.), Cognitive theon (Vol. 1), Potomac, Md: Eslbaum Associates. 1975

Cooper. W. E.. \& Blumstein, S. A "labial" feature analyzer in speech perception. Perception \& Psychophysics, 1974, 15, 590-600.

Cooper, W. E., \& Nagen, R. M. Perceptuo-motor adaptation to speech: An analysis of bisyllabic utterances and a neural model. Journal of the Acoustical Society of America, 1975, $58,256-265$

Eimas, P. D., Cooper, W. E., \& Corbit, J. D. Some properties of linguistic feature detectors. Perception \& Psychophysics, 1973, 13, 247.252.

Ermas, P. D., \& Corbit, J. 1). Selective adaptation of linguistic feature detectors. Cognitive Psychology, 1973, 4, 99.109.

Liberman, A. M., Cooper, F. S., Shankweiler, D. P., \& Studdert-Kennedy, M. Perception of the speech code. Psycholugical Review, 1967, 74, 431-461.

Milfer, J. L. Properties of feature detectors for speech: Evidence from the effects of selective adaptation on dichotic listening. Perception \& Psychophysics, 1975, 18, 389.397.
PisonI, D. B. Dichotic listening and processing phonetic features. In F. Restle, R. M. Shitfrin, N. J. Castellan, H. Lindman, \& D. B. Pisoni (Eds.), Cognitive theory (Vol. I). Potomac, Md: Erlbaum Associates, 1975.

Pisoni, D. B., \& SAwusch, J. R. Some stages of processing in speech perception. In A. Cohen \& S. Nooteboom (Eds.), Structure and process in speech perception. Heidelberg: Springer-Verlag, 1975.

Pisoni, D. B., \& TAsH, J. B. Auditory property detectors and processing place features in stop consonants. Penception \& Psychophysics, 1975, 18, 401-408.

TARTTER, V. C., \& EIMAS, P. D. The role of auditory and phonetic feature detectors in the perception of speech. Perception \& Psychophysics, 1975, 18, 293-298.

(Received for publication January 15, 1976; revision received April 25,1976 .) 